\title{
FUNDUS FLUORESCEIN ANGIOGRAPHY PATTERNS OF BRANCH RETINAL VEIN OCCLUSION AT A TERTIARY CARE CENTRE- A CROSS-SECTIONAL STUDY
}

\author{
Murali Krishna Damdamraju1, Jagannath Challa², Sandhya C. S3, Sindhura Gajula ${ }^{4}$ \\ ${ }^{1}$ Assistant Professor, Department of Ophthalmology, Sri Venkateswara Medical College, Tirupati, India. \\ ${ }^{2}$ Associate Professor, Department of Ophthalmology, Sri Venkateswara Medical College, Tirupati, India. \\ ${ }^{3}$ Professor and HOD, Department of Ophthalmology, Sri Venkateswara Medical College, Tirupati, India. \\ ${ }^{4}$ Senior Resident, Department of Ophthalmology, Sri Venkateswara Medical College, Tirupati, India.
}

\begin{abstract}
BACKGROUND
ABSTRACT

Retinal vein occlusion (RVO) is a significant cause of visual loss, second to diabetic retinopathy among the retinal vascular disorder and is a disease of the elderly, most commonly occurring between 60 and 70 years of age causing visual morbidity which has got public health significance.

The objective of our study is to study the Fluorescein Angiography patterns (FFA) characteristics of macula in Branch retinal vein occlusion (BRVO) at a tertiary care hospital.
\end{abstract}

\section{MATERIALS AND METHODS}

A descriptive study was conducted at Department of Ophthalmology, Sri Venkata Ramnarayan Ruia Government General Hospital (SVRRGGH) attached to Sri Venkateswara Medical College (SVMC), Tirupati from December 2012 to November 2016.64 eyes of 64 patients with BRVO fulfilling the inclusion criteria were examined as per protocol and results were analysed.

\section{RESULTS}

Majority of the patients were in the age group of $60-70$ years accounting for $41 \%$ and 38 (59\%) were males and 26 (41\%) were females. $32(50 \%)$ patients had capillary non-perfusion areas of $>1 / 2$ DD but less than 5 DD. Perifoveal capillary network distortion of $>3$ clock hours was noted in 44 (68.75\%) of the cases. Cystoid macular oedema was seen in 24 (37.5\%) of cases.

\section{CONCLUSION}

Fundus Fluorescein Angiography is an indispensable tool and is useful in differentiating ischaemic from non-ischaemic macular oedema which plays a major role in plan of treatment.

\section{KEYWORDS}

Branch Retinal Vein Occlusion (BRVO), Cystoid Macular Oedema (CME), Fundus Fluorescein Angiography (FFA), Perifoveal Capillary Network Distortion (PCND).

HOW TO CITE THIS ARTICLE: Damdamraju MK, Challa J, Sandhya CS, et al. Fundus fluorescein angiography patterns of branch retinal vein occlusion at a tertiary care centre- A cross-sectional study. J. Evolution Med. Dent. Sci. 2017;6(89):6175-6178, D0I: $10.14260 /$ jemds/2017/1342

\section{BACKGROUND}

Retinal vein occlusion (RVO) is a significant cause of visual loss, second to diabetic retinopathy among the retinal vascular disorders. They are of two types: Central Retinal Vein Occlusion (CRVO) and Branch Retinal Vein Occlusion (BRVO). BRVO can be considered a major BRVO or a macular BRVO. Branch retinal vein occlusion is defined as a segmental intraretinal haemorrhage not exceeding the midline caused by obstruction in the vein draining the corresponding retinal area.(1) The prevalence of RVO has been shown to vary from $0.6 \%$ to $1.1 \%$. BRVO constitutes about $2 / 3^{\text {rd }}$ of these cases with the remaining $1 / 3^{\text {rd }}$ being CRVO. BRVO is an ocular condition that has public health significance. Recognition of retinal vein occlusions is of particular importance because

'Financial or Other Competing Interest': None.

Submission 24-10-2017, Peer Review 05-11-2017,

Acceptance 07-11-2017, Published 13-11-2017.

Corresponding Author:

Dr. Jagannath Challa,

Associate Professor,

Department of Ophthalmology,

SVRRGG Hospital and

Sri Venkateswara Medical College,

Tirupati-517501, India.

E-mail: jagannathchalla@gmail.com

DOI: $10.14260 /$ jemds $/ 2017 / 1342$

\section{(c) (i) $\odot$}

their complications and significant visual morbidity. A 10 years' incidence rate of $1.2 \%$ and $0.4 \%$ for BRVO and CRVO, respectively was seen in a population-based study of 3654 participants in Australia.(2) In the Beaver Dam Eye study, RVO accounted for $12 \%$ of cases with visual acuity worse than $20 / 200$.(3) In India, RVO was detected in $0.8 \%$ of adults, and BRVO was approximately seven times more common than CRVO with a prevalence of $0.66 \% \pm 0.12 \%$ per subject. ${ }^{(4)}$ Risk factors associated with retinal vein occlusion include Diabetes mellitus, Hypertension, Glaucoma, Hyperviscosity, Hyperhomocysteinaemia and advanced age. Macular oedema is a common sequela and major cause of visual disturbance associated with BRVO. It is usually a unilateral condition. Fundus Fluorescein Angiography is an invasive imagining modality which evaluates the angiographic pattern of choroidal and retinal vasculature. It helps in identifying macular oedema, Capillary non-perfusion areas (CNP), Perifoveal capillary network distortion (PCND) and differentiates ischaemic type from non-ischaemic type. Due to these reasons, the present study was taken up.

\section{Objectives}

The purpose of this study is to diagnose macular oedema in eyes with BRVO, location and pattern of the occlusion effects, the clinical picture in BRVO, fundus fluorescein angiography 
(FFA) patterns, extent of macular oedema and ischaemia. Its role in BRVO is in the evaluation of retinal capillary nonperfusion areas, posterior segment neovascularisation, and macular oedema.

\section{MATERIALS AND METHODS}

The present study is a descriptive study conducted in the Outpatient Department and wards of Department of Ophthalmology, Sri Venkata Ramnarayan Ruia Government General Hospital (SVRRGGH) attached to Sri Venkateswara Medical College (SVMC), Tirupati from December 2012 to November 2016.

\section{Patients and Methods}

64 patients of Branch retinal vein occlusion with decreased visual acuity attending the Department of Ophthalmology, SVRRGGH, Tirupati from December 2012 to November 2016 were included. Written and informed consent was obtained from all patients included in the study. Detailed history was taken regarding the demographics, chief complaints including the duration of problem, presence of systemic diseases like hypertension, diabetes mellitus and hyperlipidaemia. Visual acuity and best corrected visual acuity in all patients recorded using Snellen's chart. All patients were examined by using slit-lamp examination, recorded intraocular pressure with Goldman applanation Tonometer, Gonioscopy. Detailed fundus examination was done with 90 D lens. Fundus Fluorescein Angiography (FFA) done in all patients. Optical coherence tomography was done wherever necessary. Systemic risk factors evaluated in all patients.

\section{Inclusion Criteria}

Subjects with BRVO Fulfilling the following Criteria were Included in the Study-

1. Best Corrected Visual Acuity of less than 6/12.

2. Macular involvement secondary to vein occlusion on clinical examination.

3. Pupillary dilatation and subject cooperation sufficient for adequate fundus photography and FFA.

\section{Exclusion Criteria}

1. Hazy media interfering with FFA interpretation.

2. Patients not willing to participate in the study.

\section{Procedure}

Fundus photographs and Fundus fluorescein angiography were performed with Digital fundus cameras (Topcon, TRC 50DX, Topcon Corporation, Tokyo, Japan and CF-1 CameraCanon Inc, Tokyo, Japan respectively). All OCT scans were performed using the Spectral Domain Optical Coherence Tomography (SD OCT). (RTVue RT 100, Software version 6.1.0.4, Optovue Inc, Fermont, CA, USA.)

Patient is instructed to come for angiography with 3 hours fasting period before the procedure to reduce the incidence of nausea and vomiting. Patient is advised to come along with an attendant. After taking written informed consent which also includes permission for using the details in medical journals and books, FFA was done.

Colour, red-free photography was taken. Control picture of the uninvolved eye was taken first, then the involved eye concentrating on the area of interest. Fluorescein angiography with fluorescein filters was started with flash intensity set for it.

$2 \mathrm{~mL}$ of $25 \%$ fluorescein dye followed by $5 \mathrm{~mL}$ of distilled water was injected into the antecubital vein after simultaneously starting timer from zero. As the normal arm to retina circulation time is $10-12$ seconds, the first photograph was taken 10-12 seconds after the injection of dye.

Photographs were then taken at an interval of 1-2 seconds for 10 seconds, 2 seconds interval for 30 seconds. Late photographs were then taken after 3,5 and 10 minutes.

The following Findings were Noted and Evaluated in FFA

- Capillary non-perfusion area.

- Perifoveal capillary distortion.

- Serous detachment of retina.

- Cystoid macular oedema.

\section{RESULTS}

In the present study, majority of the patients were in the age group of $60-70$ years accounting for $41 \%$ and 38 (59\%) were males and 26 (41\%) were females. (Chart 1) The male: female ratio was 1.5:1. In $42(66 \%)$ cases, superotemporal quadrant was involved and inferotemporal quadrant involved in $22(34 \%)$ cases. (Table 1$)$. The most common risk factor was found to be hypertension in 38 cases (59.37\%) followed by diabetes in 22 cases (34.37\%). Both hypertension and diabetes were present in 16 (25\%) cases. (Table 2).

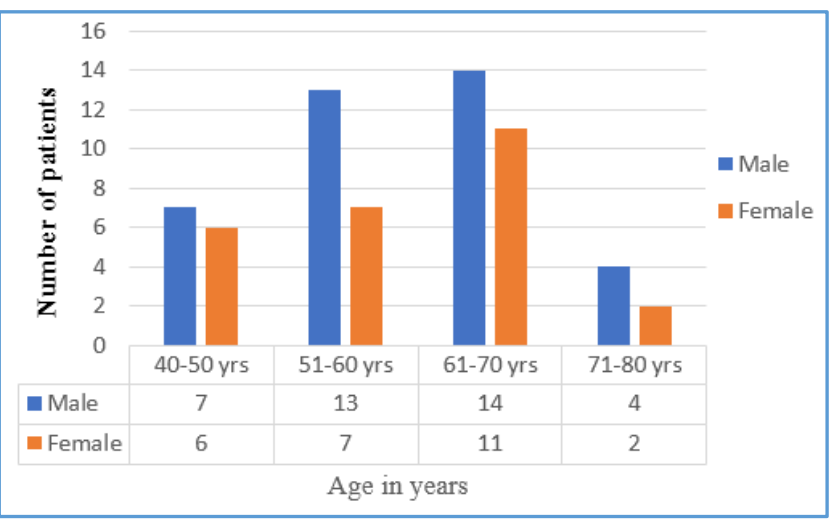

Chart 1. Showing Age wise and Sex wise Distribution

\begin{tabular}{|c|c|c|c|}
\hline Quadrant & Superotemporal & Inferotemporal & Total \\
\hline Number & 42 & 22 & 64 \\
\hline$\%$ & 66 & 34 & 100 \\
\hline \multicolumn{3}{|c|}{ Table 1. Showing Quadrant Distribution } \\
\hline
\end{tabular}

\begin{tabular}{|c|c|c|c|c|}
\hline 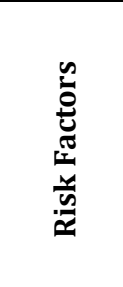 & 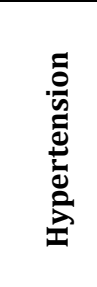 & 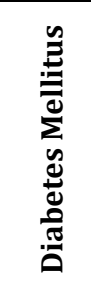 & 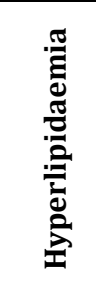 & 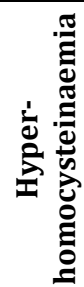 \\
\hline Number & 38 & 22 & 12 & 16 \\
\hline$\%$ & 59.37 & 34.37 & 18.75 & 25 \\
\hline \multicolumn{5}{|c|}{ Table 3. Showing Risk Factors in BRVO } \\
\hline
\end{tabular}


In the present study, 32 (50\%) patients had capillary nonperfusion areas of $>1 / 2$ DD but less than 5 DD. CNP areas $>5$ DD were seen in $24(37.5 \%)$ patients. These patients require careful monitoring as they have higher chances of developing neovascularisation of optic disc or elsewhere.

In the present study, perifoveal capillary network distortion of $>3$ clock hours was noted in 44 (68.75\%) of the cases. Neovascularisation of the disc (NVD) and neovascularisation elsewhere (NVE) were seen in 2 patients as evidenced by the late leakage of the dye from disc. Cystoid macular oedema was seen in $24(37.5 \%)$ of cases. No case of serous retinal detachment was noted. (Table 4).

\begin{tabular}{|c|c|c|c|}
\hline $\begin{array}{c}\text { FFA } \\
\text { Findings }\end{array}$ & CNP>1/2 DD & PCN Distortion & CME \\
\hline No & 56 & 44 & 24 \\
\hline$\%$ & 87.5 & 68.75 & 37.5 \\
\hline \multicolumn{3}{|c|}{ Table 4. Showing FFA Findings } \\
\hline
\end{tabular}

\section{DISCUSSION}

Retinal vein occlusion was first described in 1855 by Liebreich as retinal apoplexy and in 1878 was recognised as thrombosis by Michel.(5) Venous occlusive diseases are among the most common retinal diseases. Retinal vein occlusion including both central retinal vein occlusion (CRVO) and branch retinal vein occlusion (BRVO) represents the largest group of vascular retinal affections after arteriosclerotic hypertensive changes and diabetic retinopathy and a frequent cause of macular oedema.(6) The prognostic factors in these cases have been closely studied. From these investigations, suggested factors with prognostic significance have been the site of occlusion, extent of the macular oedema, disruption of the foveal arcade and serous retinal detachment.

Age wise distribution showed that $8(18.8 \%)$ patients between 41-50 years, 20 (31.25) patients between 51-60 years, $26(40.6 \%)$ patients between $61-70$ years and $6(9.3 \%)$ patients between 71-80 years age group were affected. The most common group affected in our study was 61-70 years. This was in accordance to the study on retinal vein occlusion by Hayreh et al., where in the onset of the first episode of RVO occurred anywhere between the ages of 14 and 92 years, with $51 \%$ having the first episode at age $\geq 65$ years.(1) The mean age in the study group was $60.1 \pm 5$ years. This was the same as the Hisayama study in which the mean age group of the patients was $67 \pm 7$ years. ${ }^{(7)}$

In the present study, branch retinal vein occlusion was more common among males (38 cases, 59.4\%) than females $(26,40.6 \%)$. The male: female ratio being $1.5: 1$. This closely corresponded to Hayreh et al study in which male: female ratio was 1.2:1.(1) In the present study, the most common risk factor was found to be hypertension in 38 (59.37\%) cases. This was similar to that found in Hayreh et al and(8) Eye Disease Case-Control Study.(9) In the present study, the prevalence of diabetes mellitus is $34.37 \%(22 / 64)$. This was almost similar to that found by Hayreh et al.(8) Many studies have shown association between retinal vein occlusion and hyperlipidaemia.(9),(10),(11) In the present study, the prevalence of hyperlipidaemia in patients with BRVO was $18.75 \%$ $(12 / 64)$ which was similar to other studies. In the present study, hyperhomocysteinaemia was present in 16 (25\%) patients. The superotemporal quadrant was most commonly involved in 42 (65.6\%) cases followed by the inferotemporal quadrant in $22(34.4 \%)$ cases in the present study.

This was in close relation to a number of studies which show that the BRVO occurs most frequently in the superotemporal quadrant.(12) This may be due to the frequent $\mathrm{AV}$ crossings in the superotemporal quadrant and the high frequency of the retinal arteriole found lying anterior to the vein toward the vitreous cavity. The superotemporal quadrant occlusion causes more macular oedema than vein occlusion elsewhere and are more symptomatic leading to a presentation bias. (Fig. 1).

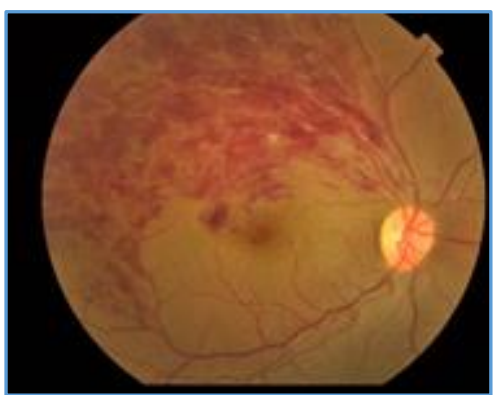

Figure 1A. Colour Fundus Image showing Superotemporal BRVO Involving Macula

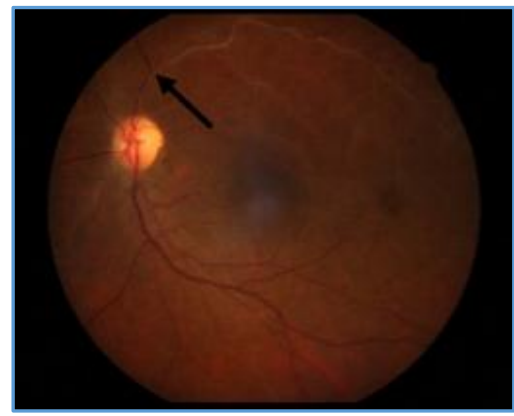

Figure 1B. Colour Fundus Photo showing Site of Occlusion in BRVO (Black Arrow)
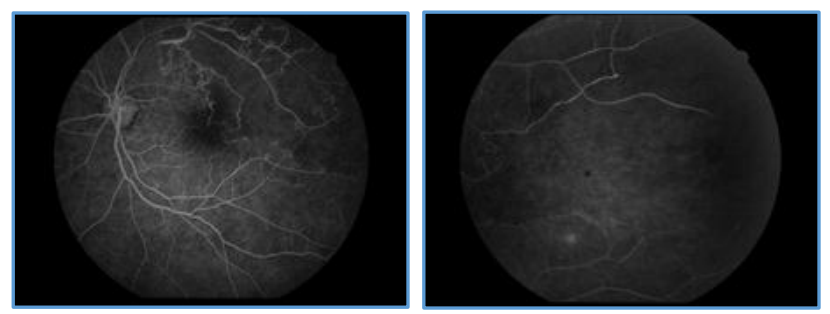

Figure 2A 2B. FFA image (Late Phase) Showing CNP Areas $>5$ DD

In the present study, 32 (50\%) patients had capillary nonperfusion areas of $>1 / 2$ DD but less than 5 DD. CNP areas $>5$ DD were seen in $24(37.5 \%)$ patients. (Fig. 2A, B) These patients require careful monitoring as they have higher chances of developing neovascularisation of optic disc or elsewhere.

In the present study, perifoveal capillary network distortion of $>3$ clock hours was noted in 44 (68.75\%) of the cases (Fig 3). Neovascularisation of the disc (NVD) and neovascularisation elsewhere (NVE) were seen in 2 patients as evidenced by the leakage of the dye from disc. Cystoid macular oedema was seen in $24(37.5 \%)$ of cases. No case of serous retinal detachment was noted. (Fig. 4). 


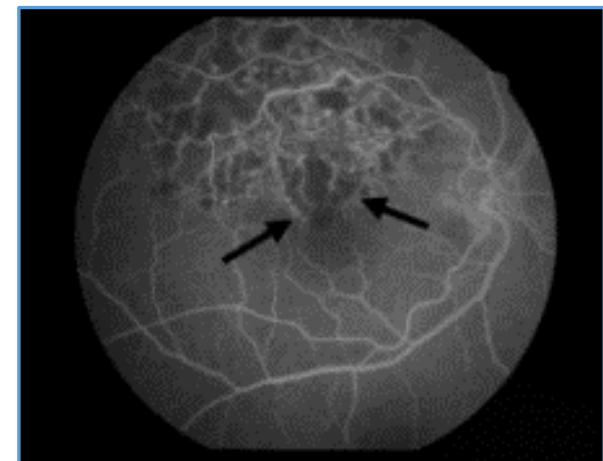

Figure 3. FFA Late Venous Phase showing PCN Distortion more than 3 Clock Hours (Between Black Arrows)

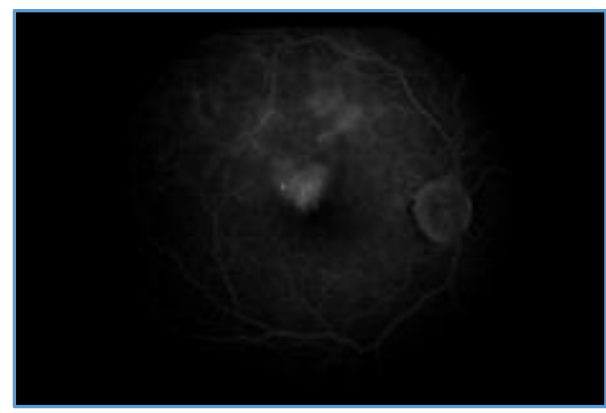

Figure 4. FFA Late Phase showing Cystoid Macular Oedema

The results were also similar to the Lang et al study (1992), which showed that cystoid macular oedema was present in $46.4 \%$ cases.(13) However, this was not in accordance with the Spaide et al, study (2003) where capillary non-perfusion was evident in 7 patients (50\%), and SRD was less common in patients with capillary nonperfusion than in those without capillary non-perfusion. The perifoveal capillary network was involved in 2 (14.28\%) patients, and the limited number of patients involved obviated any statistical evaluation. 4 (28.5\%) eyes were found to have cystoid macular oedema by fluorescein angiography.(14) The difference in results were probably due to the small sample size (14 cases) in their study compared to the present study (32 cases) and it needs further evaluation.

\section{Limitations}

Interpretation of FFA findings are difficult when done in patients with haemorrhages in the involved quadrant in early stages of BRVO (Fig. 5). FFA cannot be done in persons of known hypersensitivity to the dye.

\section{CONCLUSION}

In the present study, a detailed evaluation of patients with BRVO was done clinically and by FFA for early diagnosis with high sensitivity to detect the cause for decreased visual acuity. The most common complication of BRVO is cystoid macular oedema and thus it is essential to detect it at the earliest. FFA is a useful imaging technique available at present for objective assessment of the types, extent of CME, macular ischaemia and followup of cases with CME. Although FFA is an invasive procedure, it is very useful in differentiating ischaemic from non-ischaemic macular oedema. It is also useful in differentiating NVD from collaterals. It helps in identifying patients who require longterm follow-up.

\section{REFERENCES}

[1] Hayreh SS. Prevalent misconceptions about acute retinal vascular occlusive disorders. Progress in Retinal and Eye Research 2005;24(4):493-519.

[2] Mitchell P, Smith W, Chang A. Prevalence and associations of retinal vein occlusion in Australia. The Blue Mountains Eye Study. Arch Ophthalmol 1996;114(10):1243-7.

[3] Klein R, Moss SE, Meuer SM, et al. The 15-year cumulative incidence of retinal vein occlusion: the Beaver Dam Eye Study. Arch Ophthalmol 2008;126(4):513-8.

[4] Jonas JB, Nangia V, Khare A, et al. Prevalence and associations of retinal vein occlusions: The Central India Eye and Medical Study. Retina 2013;33(1):152-9.

[5] Hoerauf H. Branch retinal vein occlusion. In: Joussen AM. edr. Retinal vascular disease. Berlin, Heidelberg: Springer-Verlag 2007:467-501.

[6] Finkelstein D. Ischemic macular edema. Recognition and favorable natural history in branch vein occlusion. Arch Ophthalmol 1992;110(10):1427-34. http://www.ncbi.nlm.nih.gov/pubmed/1417544

[7] Yasuda M, Kiyohara Y, Arakawa S, et al. Prevalence and systemic risk factors for retinal vein occlusion in a general Japanese population: the Hisayama study. Invest Ophthalmol Vis Sci 2010;51(6):3205-9.

[8] Hayreh SS, Zimmerman B, McCarthy MJ, et al. Systemic diseases associated with various types of retinal vein occlusion. Am J Ophthalmol 2001;131(1):61-77.

[9] Risk factors for central retinal vein occlusion. The Eye Disease Case-Control Study Group. Arch Ophthalmol 1996;114(5):545-54.

[10] Cheung N, Klein R, Wang JJ, et al. Traditional and novel cardiovascular risk factors for retinal vein occlusion: the multiethnic study of atherosclerosis. Invest Ophthalmol Vis Sci 2008;49(10):4297-302.

[11] Risk factors for branch retinal vein occlusion. The Eye Disease Case-control Study Group. Am J Ophthalmol 1993;116(3):286-96.

[12] Weinberg D, Dodwell DG, Fern SA. Anatomy of arteriovenous crossings in branch retinal vein occlusion. Am J Ophthalmol 1990;109(3):298-302.

[13] Lang GE, Freissler K. Clinical and fluorescein angiography findings in patients with retinal vein occlusion. A unicenter study of 211 patients. Klin Monbl Augenheilkd 1992;201(4):234-9.

[14] Spaide RF, Lee JK, Klancnik JK, et al. Optical coherence tomography of branch retinal vein occlusion. Retina 2003;23(3):343-7. 